ACCEPTED MANUSCRIPT

\title{
Dopamine-functionalized graphene oxide as a high-performance material for biosensing
}

To cite this article before publication: Fabio Vulcano et al 2020 2D Mater. in press https://doi.org/10.1088/2053-1583/ab734f

\section{Manuscript version: Accepted Manuscript}

Accepted Manuscript is "the version of the article accepted for publication including all changes made as a result of the peer review process, and which may also include the addition to the article by IOP Publishing of a header, an article ID, a cover sheet and/or an 'Accepted Manuscript' watermark, but excluding any other editing, typesetting or other changes made by IOP Publishing and/or its licensors"

This Accepted Manuscript is @ 2020 IOP Publishing Ltd.

During the embargo period (the 12 month period from the publication of the Version of Record of this article), the Accepted Manuscript is fully protected by copyright and cannot be reused or reposted elsewhere.

As the Version of Record of this article is going to be / has been published on a subscription basis, this Accepted Manuscript is available for reuse under a CC BY-NC-ND 3.0 licence after the 12 month embargo period.

After the embargo period, everyone is permitted to use copy and redistribute this article for non-commercial purposes only, provided that they adhere to all the terms of the licence https://creativecommons.org/licences/by-nc-nd/3.0

Although reasonable endeavours have been taken to obtain all necessary permissions from third parties to include their copyrighted content within this article, their full citation and copyright line may not be present in this Accepted Manuscript version. Before using any content from this article, please refer to the Version of Record on IOPscience once published for full citation and copyright details, as permissions will likely be required. All third party content is fully copyright protected, unless specifically stated otherwise in the figure caption in the Version of Record.

View the article online for updates and enhancements. 


\title{
Dopamine-functionalized graphene oxide as a high-performance material for biosensing
}

\author{
Fabio Vulcano ${ }^{1,2}$, Alessandro Kovtun ${ }^{2}$, Cristian Bettini ${ }^{2}$, Zhenyuan Xia ${ }^{3,2}$, Andrea \\ Liscio ${ }^{4}$, Fabio Terzi ${ }^{1}$, Aranzazu Heras ${ }^{5}$, Alvaro Colina ${ }^{5}$, Barbara Zanfrognini, ${ }^{2}$ \\ Manuela Melucci ${ }^{2,}{ }^{*}$, Vincenzo Palermo ${ }^{2,3,{ }^{*}}$, Chiara Zanardi ${ }^{1,2,{ }^{*}}$ \\ ${ }^{1}$ Department of Chemical and Geological Sciences, Università di Modena e Reggio Emilia, Via G. \\ Campi 103, 41125 Modena, Italy \\ ${ }^{2}$ Institute of Organic Synthesis and Photoreactivity (ISOF), National Research Council of Italy (CNR), \\ via P. Gobetti 101, 40129 Bologna, Italy. \\ ${ }^{3}$ Department of Industrial and Materials Science, Chalmers University of Technology, SE-412 96 \\ Gothenburg, Sweden \\ ${ }^{4}$ Institute of Microelectronics and Microsystems (IMM), National Research Council of Italy (CNR), via \\ del Fosso del Cavaliere 100, 00133 Rome, Italy \\ ${ }^{5}$ Department of Chemistry, Universidad de Burgos, Pza Misael Bañuelos s/n, E-09001 Burgos, Spain
}

E-mail: manuela.melucci@isof.cnr.it; vincenzo.palermo@isof.cnr.it; chiara.zanardi@unimore.it

Received xxxxxx

Accepted for publication $\mathrm{xxxxxx}$

Published xxxxxx

\begin{abstract}
We describe a nanocomposite material for the electrochemical detection of $\beta$-nicotinamide adenine dinucleotide (NADH), a coenzyme involved in redox reactions of all living cells and in the detection of many organic species by electrochemical biosensors. The composite is made of nanosheets of electrochemically exfoliated graphene oxide (EGO) covalently functionalized with dopamine (DP) molecules. The $\mathrm{EGO}_{\mathrm{DP}}$ material finally obtained is rich of 1,2-dihydroxyphenyl moieties and is able to detect NADH at a particular low potential value with higher sensitivity with respect to pristine EGO.

To study the effectiveness of 1,2-dihydroxyphenyl moieties in inducing electrocatalytic oxidation of NADH, we combined standard voltammetric techniques with UV-Vis absorption spectroelectrochemistry, which allowed us to measure the variations in composition occurring at the electrode|solution interface, i.e. to measure the consumption rate of NADH.

Spectroelectrochemical tests performed by polarising the electrode at a fixed potential value were finally used to compare the performance of $\mathrm{EGO}_{\mathrm{DP}}$ with both $\mathrm{EGO}$ and EGO-DP blend (MIX) for the detection of NADH. The covalently functionalized EGO (EGO $\left.{ }_{\mathrm{DP}}\right)$ shows sensitivity to NADH up to $300 \mathrm{M}^{-1}$, around $180 \%$ and $140 \%$ better than either pristine EGO or MIX, respectively.
\end{abstract}

Keywords: graphene oxide, spectroelectrochemistry, NADH, chemical functionalization, 1,2-dihydroxyphenyl moieties

\section{Introduction}

Graphene is a carbon allotrope possessing a bidimensional structure formed by $\mathrm{sp}^{2}$-hybridized carbon atoms and constituting the building block of different carbon allotropes, such as 0D fullerenes, 1D carbon nanotubes and 3D graphite. It possesses quite peculiar properties, like high surface area $\left(>2600 \mathrm{~m}^{2} / \mathrm{g}\right.$ for single-layer graphene), excellent electron mobility and mechanical resistance. Due to 


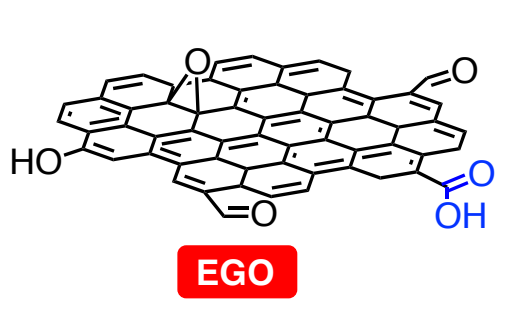

i) EDAC, NHS, DMF

ii) $\mathrm{DP}, \mathrm{NaHCO}_{3}$ sat

\section{a)}

b)

Scheme 1. Synthetic route to $\mathrm{EGO}_{\mathrm{DP}}$ (path a) and to MIX (path b). a) C-N coupling reaction by EDAC/NHS activation under $\mathrm{N}_{2}$ atmosphere, starting from $\mathrm{a} \approx 3 \mathrm{mg} \mathrm{mL}^{-1}$ EGO solution in DMF and b) reaction carried out without activation of the carboxylic groups, starting from $\mathrm{a} \approx 3 \mathrm{mg} \mathrm{mL}^{-1} \mathrm{EGO}$ solution in DMF. In this conditions epoxide ring opening reaction by amine groups of DP could occur (as indicated in the magenta box) as also suggested by XPS data showing a slight decrease in C-O-C bonds only for the MIX (and not for $\mathrm{EGO}_{\mathrm{DP}}$, Fig. 1b). Details on the synthesis are reported in the experimental section 2.1.

all these reasons, this material is nowadays widely studied for a number of different applications [1-5].

Graphene sheets are scarcely soluble and can be processed only in aggressive solvents or using surfactants. For many applications, instead, an oxidized water-soluble derivative of graphene is generally preferred, namely graphene oxide (GO). It can be produced by chemical oxidation of graphite to graphite oxide and subsequent exfoliation. This is considered the most economical way to obtain mass production of monoatomic carbon sheets with high yield. The resulting material is a highly defective $\mathrm{sp}^{2}$-hybridized structure, featuring a rich surface chemistry with different oxygenated functions, namely epoxide, hydroxyl, carbonyl and carboxyl groups [6,7].

In previous works, we reported the advantage in the use of an electrochemically exfoliated graphene oxide (EGO) [8]. It contains a suitable number of oxidized moieties making it electrically conductive and able to interact with the analytes in solution, at the same time [7]. In particular, the hydroxyl and carboxylic groups confer to the material peculiar reactivity with respect to species in solution: these moieties show electrocatalytic properties toward some target analytes, decreasing the overpotential at which the charge transfer process related to their oxidation takes place [7]. Besides, they can also act as the binding site for a further functionalization of the carbon-based nanosheets [9-11], aimed at modulating the properties of the material. All these properties favorably improve the performance of the material when used in electrochemical sensors for the detection of species of biological and forensic interest $[7,12]$.

Among different species studied in the frame of sensors, peculiar attention should be paid to the detection of $\beta$ - nicotinamide adenine dinucleotide (NADH), a chargetransfer mediator of many enzymatic reactions. Abnormal levels of NADH in living cells, as example, can indicate altered status of health [13] and can be monitored by electrochemical sensors [14,15]. Additional possible applications of NADH for sensing are manifold, because it can also be combined with specific NADH-dependent enzymes to indirectly detect many chemical species produced or consumed by such enzymes [16-19], e.g. lactate by lactate dehydrogenase [20,21], glycerol by glycerol dehydrogenase [22,23], glucose by glucose dehydrogenase $[21,24]$ and alcohols by alcohol dehydrogenase [25].

We recently demonstrated that the hydroxyl moieties on the graphene nanosheets are responsible for the significant anticipation of the NADH oxidation process from +0.49 to $+0.18 \mathrm{~V} v s$. $\mathrm{Ag} / \mathrm{AgCl}$ when passing from bare to reduced GO modified electrodes [7]. The shifting of the electrochemical response at less positive potentials is considered as an added value to the performance of the sensing element, because it may allow to discriminate the NADH signal from responses coming from different species in solution.

The above-mentioned hydroxyl moieties derive from the disruption of the honeycomb-structure of pure carbon nanosheets and actually consist of electroactive hydroxyphenyl residues. They can catalyze the oxidation of $\mathrm{NADH}$ thanks to a well-known electron-hopping process [26-28]. For this reason, it is very likely that the sensor sensitivity at this quite low overpotential is directly related to the number of $-\mathrm{OH}$ groups on the electrode. However, for both electrochemically exfoliated and chemically produced $\mathrm{GO}$, it is not possible to finely tailor the degree of $-\mathrm{OH}$ groups available on the surface. Precise positioning of 
chemical groups on graphene can be achieved by selfassembly and electrochemistry [19], but such approach is suitable only for flat, pristine graphene. Here, to control and increase the number of surface - $\mathrm{OH}$ groups of $\mathrm{GO}$, we propose an unprecedented approach, i.e. the covalent grafting of dopamine moieties (DP, Scheme 1, path a). DP is a wellknown molecule in biochemistry for its role in regulating several biological processes. It features electroactive 1,2dihydroxyphenyl groups on its aromatic ring and an amine moiety exploitable to form covalent amidic bonds.

Grafting of DP on EGO was obtained by $\mathrm{C}-\mathrm{N}$ coupling between carboxylic residues, activated by Nhydroxysuccinimide (NHS) and 1-ethyl-3(dimethylaminopropyl)carbodiimide hydrochloride (EDAC), and amine moieties of DP (Scheme 1, path a). In such conditions epoxide ring opening could also occur, achieving beneficial maximization of the degree of functionalization with amines [30]. The so obtained $\mathrm{EGO}_{\mathrm{DP}}$ was characterized in comparison to a reference system consisting of a mixture of EGO nanosheets and DP molecules, hereafter defined as MIX (Scheme 1, path b), which was obtained in the same experimental conditions exploited for the preparation of $E O_{D P}$ but without activation of the carboxylic acids of EGO.

We defined the effectiveness of 1,2-dihydroxyphenyl residues in the detection of NADH by combining electrochemical results with that of UV-Vis adsorption spectra simultaneously collected, i.e. by performing spectroelectrochemical experiments in parallel configuration. This in-situ technique allows the correlation between the electrochemical response obtained at defined potential values, and the spectral changes occurring in $a \approx 100 \mu \mathrm{m}$ thick area of solution adjacent to the working electrode surface [31-33]. Similar spectroelectrochemical experiments allowed us to test the highest efficiency of $\mathrm{EGO}_{\mathrm{DP}}$ with respect to pristine EGO and MIX material for $/$ the spectroelectrochemical detection of NADH.

\section{Experimental}

\subsection{Synthesis of EGO-based materials}

EGO was prepared from graphite flakes (Sigma Aldrich, $99 \%$ pure, $<150 \mathrm{~mm}$ ) as reported in ref. [8,34]. DP, EDAC and NHS were purchased from Sigma Aldrich and used without further purification.

$\mathrm{EGO}_{\mathrm{DP}}$ (Scheme 1, path a) was obtained starting from EGO $(60 \mathrm{mg})$ dispersion in DMF $(20 \mathrm{~mL})$, achieved after 24 $\mathrm{h}$ of sonication. NHS $(207 \mathrm{mg}, 1.8 \mathrm{mmol})$ and then EDAC (346 $\mathrm{mg}, 1.8 \mathrm{mmol}$ ) were subsequently added at room temperature (RT) under $\mathrm{N}_{2}$ atmosphere; the resulting suspension was stirred at $50^{\circ} \mathrm{C}$ for $24 \mathrm{~h}$ and then at RT for 48 h. DP $(513 \mathrm{mg}, 2.7 \mathrm{mmol})$ and saturated $\mathrm{NaHCO}_{3}$ solution $(15 \mathrm{~mL})$ were then added. The mixture was stirred at $50^{\circ} \mathrm{C}$ for $8 \mathrm{~h}$ and then at RT for $16 \mathrm{~h}$. The crude material was purified by repeated washing and centrifugation steps $(18000$ rpm, 25 minutes) by using $\mathrm{mQ} \mathrm{H}_{2} \mathrm{O}, \mathrm{DMF}$, and $\mathrm{mQ} \mathrm{H}_{2} \mathrm{O}$ EtOH-acetone mixture (twice per solvent). $\mathrm{EGO}_{\mathrm{DP}}$ was finally obtained as a black powder ( $45 \mathrm{mg}$ ).

MIX (Scheme 1, path b) was obtained starting from EGO $(40 \mathrm{mg})$ dispersion in DMF $(14 \mathrm{~mL})$, achieved after $24 \mathrm{~h}$ of sonication. The dispersion was stirred for $24 \mathrm{~h}$ at $50^{\circ} \mathrm{C}$ and then for $48 \mathrm{~h}$ at RT under $\mathrm{N}_{2}$ atmosphere, in order to simulate the reaction conditions used for the synthesis of $\mathrm{EGO}_{\mathrm{DP}}$. Then DP $(342 \mathrm{mg}, 1.8 \mathrm{mmol})$ and saturated $\mathrm{NaHCO}_{3}$ solution $(10 \mathrm{~mL})$ were added. The dispersion was stirred at $50^{\circ} \mathrm{C}$ for $8 \mathrm{~h}$ and then at RT for $16 \mathrm{~h}$. The crude material was purified by repeated washing and centrifugation steps (18000 rpm, 25 minutes) by using mQ $\mathrm{H}_{2} \mathrm{O}$, DMF, and mQ $\mathrm{H}_{2} \mathrm{O}-\mathrm{EtOH}$-acetone mixture (twice per solvent). MIX was obtained as a black powder $(30 \mathrm{mg})$.

\subsection{Instrumental set-up}

ATR spectra of EGO and $\mathrm{EGO}_{\mathrm{DP}}$ were recorded with a Jasco FT/IR-4700 in the $400-4000 \mathrm{~cm}^{-1}$ spectral region.

UV-Vis spectra of the supernatants collected from centrifuged suspensions of $\mathrm{EGO}_{\mathrm{DP}}$ and MIX were recorded with a Lambda 650 UV-Vis spectrometer (Perkin-Elmer) using a quartz cuvette with a $1 \mathrm{~mm}$ optical path. The samples were obtained from centrifugation of $0.5 \mathrm{~g} \mathrm{~L}^{-1}$ suspensions of either $\mathrm{EGO}_{\mathrm{DP}}$ or MIX, stirred overnight, until reaching a transparent solution.

XPS measurements were performed with a Phoibos 100 hemispherical energy analyser (Specs), using $\mathrm{Mg} \mathrm{K \alpha}$ excitation source (XR-50, Specs) $(\hbar \omega=1253.6 \mathrm{eV})$. The Xray power was $125 \mathrm{~W}$. The base pressure in the analysis chamber was $1 \times 10^{-9}$ mbar. The C1s signal was fitted by fixing the chemical shift of different carbon groups after the subtraction of the Shirley background: aromatic carbon (C-C $\left.\mathrm{sp}^{2}, 284.4 \mathrm{eV}\right)$, aliphatic carbon $\left(\mathrm{C}-\mathrm{C} \mathrm{sp}{ }^{3}, 285.0 \mathrm{eV}\right)$, hydroxyl (C-OH, $285.7 \mathrm{eV})$, epoxy (C-O-C, $286.7 \mathrm{eV})$, carbonyl $(\mathrm{C}=\mathrm{O}, 288.0 \mathrm{eV})$ and carboxyl $(\mathrm{O}-\mathrm{C}=\mathrm{O}, 289.1 \mathrm{eV})$. The aromatic carbon $\left(\mathrm{C}-\mathrm{C} \mathrm{sp}{ }^{2}\right)$ was fitted by an asymmetric pseudo-Voigt (APV) curve. Asymmetry parameter was 0.14 , whereas all the others peak-shapes were symmetric Voigt. Deconvolving the C1s XPS survey, we calculated the O/C ratio as given by the ratio between the sum of contributions of different oxidized residues and the sum of $\mathrm{sp}^{2}$ and $\mathrm{sp}^{3}$ carbons [35]. The N1s signal was fitted by considering two components: a main peak due to substituted amino group (RNH-R) and a satellite assigned to primary amine residues (R$\mathrm{NH}_{2}$ ). The two components show different behaviours: while the main peak appeared at the same energy (i.e. $339.8 \pm 0.2$ $\mathrm{eV}$ ), the satellite presents some significant energy shifts for different materials: $401.6 \mathrm{eV}$ in $\mathrm{EGO}$ and $402.4 \mathrm{eV}$ in $\mathrm{EGO}_{\mathrm{DP}}$ $[36,37]$. EGO-based materials were analysed in the form of self-standing films, obtained after filtration of their 
suspensions in isopropyl alcohol (1 $\mathrm{mg}$ in $40 \mathrm{~mL}$ ) throughout a ceramic filter $\left(\mathrm{Al}_{2} \mathrm{O}_{3}\right)$. Further details concerning preparation of XPS samples are reported in ref. [24].

The morphology of the EGO-based materials was investigated after their deposition on an indium tin oxide (ITO) glass by a Nova NanoSEM 450 scanning electron microscope (SEM, FEI company), working in high vacuum conditions and equipped with an Energy Dispersive Spectrometer (EDS, Bruker, QUANTAX 200). ITO slides, approximately $1 \times 3 \mathrm{~cm}$, were cleaned using toluene, acetone, ultrapure water, an oxidising solution $(30 \% \mathrm{w} / \mathrm{w}$ ammonia solution and $30 \% \mathrm{w} / \mathrm{w}$ hydrogen peroxide, $3: 1$ in volume), rinsed with ultrapure water and dried using a stream of nitrogen.

Electrochemical measurements were performed in air using a computerized Autolab PGSTAT 30 (Ecochemie). Screen-printed electrodes (SPEs) were acquired from Metrohm-DropSens and consisted of a $4 \mathrm{~mm}$ diameter carbon working electrode, a graphite auxiliary electrode and an $\mathrm{Ag}$ pseudo-reference electrode. The potential of the pseudo-reference electrode was fixed by adding $0.1 \mathrm{M} \mathrm{KCl}$ to all solutions used for electrochemical tests, finally resulting shifted of $+0.02 \mathrm{~V} v s$. a standard $\mathrm{Ag} / \mathrm{AgCl} / 3 \mathrm{M} \mathrm{KCl}$ reference electrode. Modified SPEs were prepared by drop casting $6 \mu \mathrm{l}$ of $0.5 \mathrm{~g} \mathrm{~L}^{-1}$ suspensions of EGO-based materials. In particular, isopropyl alcohol was used as the solvent in the case of EGO and water for EGO deriving materials. The solvent was allowed to evaporate at RT before the use of modified-SPEs in electrochemical or spectroelectrochemical experiments.

UV-Vis absorption spectroelectrochemical experiments were performed with a customized SPELEC instrument (Metrohm-DropSens) equipped with a halogen/deuterium lamp. DropView SPELEC software (Metrohm-DropSens) was used for the real-time registration of spectra, synchronized with the electrochemical data. Two naked $100-$ $\mu \mathrm{m}$ optical fibres (Avantes), perfectly aligned with each other, were fixed to the edges of the SPE using commercial nail polish, in order to direct the light beam in the close proximity of the electrode surface. This experimental set-up was used to perform spectroelectrochemical measurements in the parallel configuration, i.e. to detect variations in the chemical composition of a portion of solution between the electrode surface and a distance of $100 \mu \mathrm{m}$ [31-33].

\subsection{Electrochemical and spectroelectrochemical measurements}

All solutions were prepared with deionized ultrapure water obtained from a Millipore DirectQ purification system provided by Millipore $\left(18.2 \mathrm{M} \Omega \mathrm{cm}\right.$ resistivity at $\left.25^{\circ} \mathrm{C}\right)$. Electrochemical and spectroelectrochemical measurements were carried out in a $0.1 \mathrm{M}$ phosphate buffer solution (PBS) and $0.1 \mathrm{M} \mathrm{KCl}$, obtained from $\mathrm{Na}_{2} \mathrm{HPO}_{4}$ (Carlo Erba, 99+\%),
$\mathrm{NaH}_{2} \mathrm{PO}_{4}$ (Carlo Erba, 98+ \%) and $\mathrm{KCl}$ (Carlo Erba, 99+ \%). $\mathrm{NADH}$ solution was prepared just before the use, starting from the commercial product (Sigma-Aldrich, 99+ \%).

The electrochemical behaviour of EGO-based materials was tested by performing 20 following voltammetric scans of modified SPEs in pure electrolyte solutions; the potential varied between -0.10 and $+0.40 \mathrm{~V}$ at $0.02 \mathrm{~V} \mathrm{~s}^{-1}$ scan rate.

The performance of the different EGO-based materials for NADH oxidation was preliminarily tested by collecting voltammetric responses in absence and in presence of 0.5 $\mathrm{mM}$ NADH solution. In this case, the potential was varied between -0.10 and $+0.60 \mathrm{~V}$ at $0.02 \mathrm{~V} \mathrm{~s}^{-1}$ scan rate.

Spectroelectrochemical experiments were performed at $\mathrm{EGO}, \mathrm{EGO}_{\mathrm{DP}}$ and MIX modified SPEs by collecting the absorbance spectra in the $280-800 \mathrm{~nm}$ interval while performing cyclic voltammetry $(\mathrm{CV})$ experiments in a 1.2 mM NADH, 0.1 M PBS (pH 7) and 0.1 M KCl solution. The potential was varied between -0.10 and $+0.60 \mathrm{~V}$ at $0.02 \mathrm{~V} \mathrm{~s}^{-1}$ for $\mathrm{EGO}_{\mathrm{DP}}$ modified SPE and at $0.005 \mathrm{~V} \mathrm{~s}^{-1}$ for pristine $\mathrm{EGO}$ modified SPE. The evolution of the UV-Vis absorption spectra were registered simultaneously to CV experiments, taking the spectrum of the starting solution at the initial open circuit potential as the reference, for each spectroelectrochemical experiment.

\subsection{Spectroelectrochemical calibrations of NADH}

Calibrations were performed in NADH solutions at concentration values ranging between 10 and $150 \mu \mathrm{M}$, by polarizing EGO, $\mathrm{EGO}_{\mathrm{DP}}$ and MIX modified-SPEs at the fixed potential of $+0.26 \mathrm{~V}$ for $30 \mathrm{~s}$ and simultaneously registering the evolution of the UV-Vis absorption spectra. From a quantitative point of view, the variation of absorbance at $350 \mathrm{~nm}$, related to the consumption of NADH, was selected. Solutions at different concentration were analysed randomly, to rule out possible memory and fouling effects deriving from the oxidation of NADH. The calibration was performed three times for each modified electrode and it was also repeated, in the case of $\mathrm{EGO}_{\mathrm{DP}}$, for two further electrodes modified under the same conditions.

\section{Results and Discussion}

\subsection{Chemical functionalization of EGO}

Our strategy was to functionalize EGO nanosheets by covalent grafting of DP molecules with the formation of amidic bonds (Scheme 1, path a). NHS/EDAC mixture in DMF was used to activate carboxylic residues mostly present at the edges of the EGO nanosheets. Alkaline conditions promoted by $\mathrm{NaHCO}_{3}$ were crucial to achieve successful coupling reaction. This was demonstrated by performing a model reaction between benzoic acid and DP and by monitoring the evolution of this reaction by mass spectrometry: successful reaction was obtained only in the 

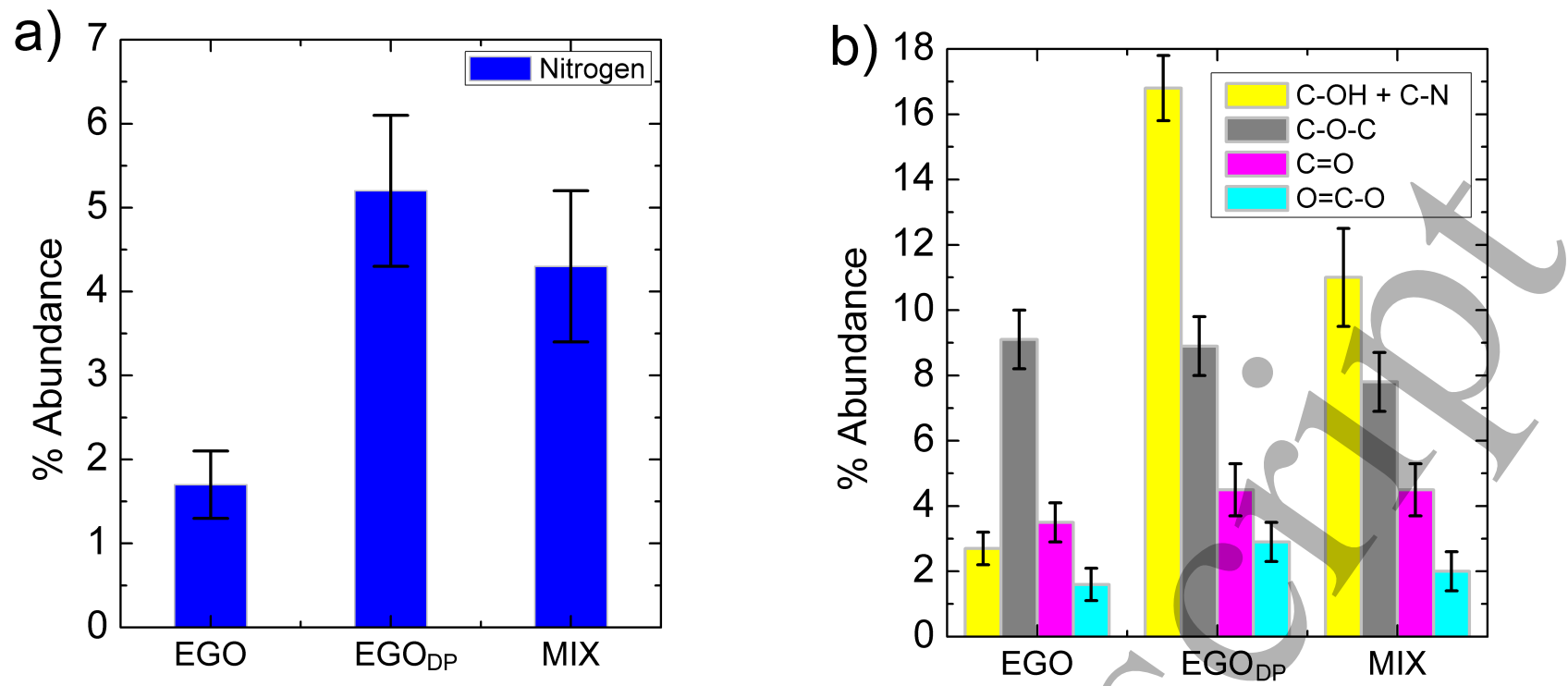

Figure 1. Abundance of (a) nitrogen and (b) different carbon-based functional groups defined by XPS analyses on the three materials. Detailed values of the abundance percentage are reported in Table S1 in ESI.

presence of $\mathrm{NaHCO}_{3}$. Since, these conditions also promote polymerization of DP to polydopamine (pDP) [38,39], the target material $\mathrm{EGO}_{\mathrm{DP}}$ was abundantly washed after the synthesis and the possible by-products of this side-reaction were taken into account in the following analyses.

In addition, since the occurrence of spontaneous interactions between EGO and DP as well as coupling by epoxide ring-opening cannot be discarded, a blend of EGO and DP, namely MIX, was also prepared for comparison (Scheme 1, path b). Interactions between these two components may occur as a consequence of $i$ ) opening of the epoxy rings by amine residue of dopamine [40], ii) electrostatic interactions between protonated amine residues of DP and negatively charges carboxylic residues, iii) $\pi-\pi$ interactions between dihydroxyphenyl rings and the aromatic carbon structure. MIX was obtained under the same experimental conditions used for the synthesis of $\mathrm{EGO}_{\mathrm{DP}}$, as to solvent, molar ratio among the different reactants, reaction time and temperature, but in absence of NHS/EDAC activating mixture. Washing and purification procedure was also the same for both $\mathrm{EGO}_{\mathrm{DP}}$ and $\mathrm{MIX}$.

\subsection{Structural and chemical characterization of functionalized EGO}

Evidence of covalent modification in $\mathrm{EGO}_{\mathrm{DP}}$ was searched by performing ATR spectroscopy, DP release tests and XPS analysis. Unfortunately, ATR spectroscopy performed on $\mathrm{EGO}$ and $\mathrm{EGO}_{\mathrm{DP}}$ did not show any difference between the two compounds (see Figure S1 in ESI).

Comparative release tests between $\mathrm{EGO}_{\mathrm{DP}}$ and MIX were performed to check the stability/strength of DP bonding/coating in the two cases. With this aim, $\mathrm{EGO}_{\mathrm{DP}}$ and
MIX samples were kept overnight in water, under continuous stirring, to favour desorption of species not covalently linked to EGO nanosheets. We then analysed with UV-Vis spectroscopy the supernatants collected, after centrifugation (see Figure S2 in ESI). It should be noted that $\mathrm{EGO}_{\mathrm{DP}}$ and MIX were purified after the synthesis by using the same protocol, i.e. reiterated washing/centrifugation steps. For MIX we observed an UV absorption peak at $c a .280 \mathrm{~nm}$, ascribable to unreacted DP molecules, only slightly evident for $\mathrm{EGO}_{\mathrm{DP}}$. In addition, it shows a broader featureless signal spanning the entire visible region, ascribable to pDP, polymerized as a side product during the grafting procedure [41]. Therefore, release tests allow us to highlight a more stable DP-EGO adduct in the case of $\mathrm{EGO}_{\mathrm{DP}}$.

XPS was used to possibly analyse the chemical composition of the nanosheets in $\mathrm{EGO}_{\mathrm{DP}}$ with respect to those of MIX. This technique is capable to detect presence of different kinds of heteroatoms and of different types of carbon chemical bonds in graphene and GO [35]. Figure 1(a) shows the abundance of nitrogen in EGO, $\mathrm{EGO}_{\mathrm{DP}}$ and MIX, as measured by N1s signal. The spectra (Figure S3 in ESI) are characterized by a main peak centred at $c a .400 \mathrm{eV}$ and ascribable to R-NH-R groups [37]. Both covalently functionalized $\mathrm{EGO}_{\mathrm{DP}}$ and MIX samples show a percentage of nitrogen significantly higher than the pristine EGO (Figure S4 in ESI). The $\approx 1.5 \%$ of $\mathrm{N}$ observed in EGO, is likely due to residual DMF molecules and impurities from the electrochemical exfoliation. The final percentages of nitrogen atoms at $\mathrm{EGO}_{\mathrm{DP}}$ and MIX were not significantly different from each other even if also MIX underwent extensive washing to remove any unbound DP, as stated above. 

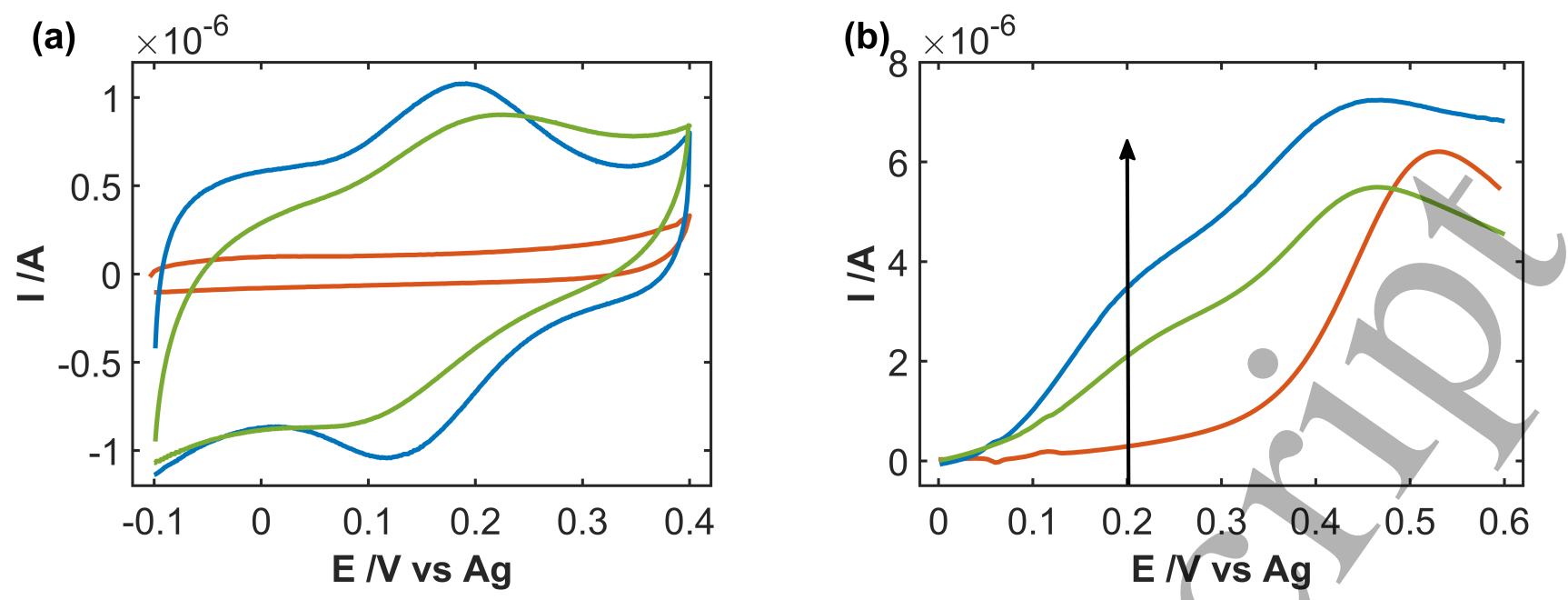

Figure 2. (a) CVs recorded at $\mathrm{EGO}$ (red line), $\mathrm{EGO}_{\mathrm{DP}}$ (blue line) and MIX (green line) modified-SPEs in $0.1 \mathrm{M}$ PBS (pH 7.0) and $0.1 \mathrm{M} \mathrm{KCl}$ after 20 potential cycles. (b) Voltammetric traces recorded at the same modified electrodes after addition of $0.5 \mathrm{mM} \mathrm{NADH}$; only the forward scan after subtraction of the relevant signal obtained in the absence of NADH is reported. Original CVs of (b) are reported in Figure S8 in ESI). Scan rate $=0.02 \mathrm{Vs}^{-1}$.

To check if there was any difference, we also analysed the XPS signal for carbon, especially to possibly detect the presence of DP molecules covalently linked to EGO nanosheets through amidic bonds. The $\mathrm{C} 1 \mathrm{~s}$ peak is typically a convolution of contributions due to different carbon functionalizations, which can be deconvolved to quantify the different chemical species present (Figure S5 and Table S1 in ESI). We see in Figure 1(b) that $E G_{D P}$ showed a signal significantly higher than the other samples in the area corresponding to C-N bonds. However, the C-N peak (285$286.5 \mathrm{eV}$ ) [37] partially overlaps the peaks due to the $\mathrm{C}-\mathrm{OH}$ and to the $\mathrm{sp}^{3}$ groups, so it was not possible to discriminate between the various species. Concurrently, spectral region ascribable to epoxide, carbonyl and carboxylic residues [34] resulted also affected by the increase of nitrogen atoms going from pristine to functionalized EGO, as observed from the quite high standard deviation associated to the different contributions (Figure 1(b)). For this reason, C1s spectra cannot be used for the quantitative study of covalent functionalization of EGO nanosheets by carboxylic residues.

Nevertheless, XPS allowed us $i$ ) to highlight a higher number of $\mathrm{OH}$ residues in $\mathrm{EGO}_{\mathrm{DP}}$, this being our main purposes, and ii) to exclude significant epoxide ring-opening in MIX since the number of epoxide residues in MIX, $\mathrm{EGO}_{\mathrm{DP}}$ and $\mathrm{EGO}$ were almost the same.

Finally we studied the morphology of $\mathrm{EGO}_{\mathrm{DP}}$ in respect to reference materials. To this aim, the $\mathrm{EGO}, \mathrm{EGO}_{\mathrm{DP}}$ or MIX solutions were drop-cast onto ITO substrates and dried in air at room temperature to study their morphology. SEM images (Figure S6 in ESI) show that all samples are composed by flakes with size $<500 \mathrm{~nm}$, even if some larger flakes are also present. The shape and dimensions of the flakes are similar for all the materials tested, indicating that the chemical functionalization does not modify their size.

SEM images were also used to look for residues and contaminants on the flakes due, as an example, to pDP formed as a side reaction during the grafting procedure. Previous work showed that pDP formed on carbon-based substrates appears as polymeric granules homogenously distributed on carbon nanosheets $[38,42]$. The images revealed that the films obtained are quite similar to each other, suggesting that the amount of pDP residual was, in any case, quite low (see also additional characterization here below).

\subsection{Electrochemical characterization of EGO modified electrodes}

Electrochemical characterizations allowed us to confirm the actual functionalization of EGO nanosheets by 1,2dihydroxyphenyl moieties, also with respect to the effect of NHS/EDAC activation mixture. Tests in presence of NADH allowed us to verify the effectiveness of these moieties in shifting the electrochemical oxidation of this species at +0.20 $\mathrm{V}$ and to compare the electrocatalytic properties of each sample.

Figure 2(a) shows the CVs of the different materials in the pure electrolyte solution. Both $\mathrm{EGO}_{\mathrm{DP}}$ and MIX feature a reversible anodic peak at $c a$. $+0.20 \mathrm{~V}$, not present in the pristine EGO, that could be ascribed to the oxidation of the 1,2-dihydroxyphenyl moieties added as a consequence of the reaction of EGO with DP. The stability of the $E \mathrm{O}_{\mathrm{DP}}$ modified electrode was obtained after $10 \mathrm{CV}$ cycles (Figure S7 in ESI), during which the current suffers a significant decrease until reaching a steady state. This is not surprising at all, since polarization of the working electrode induces 

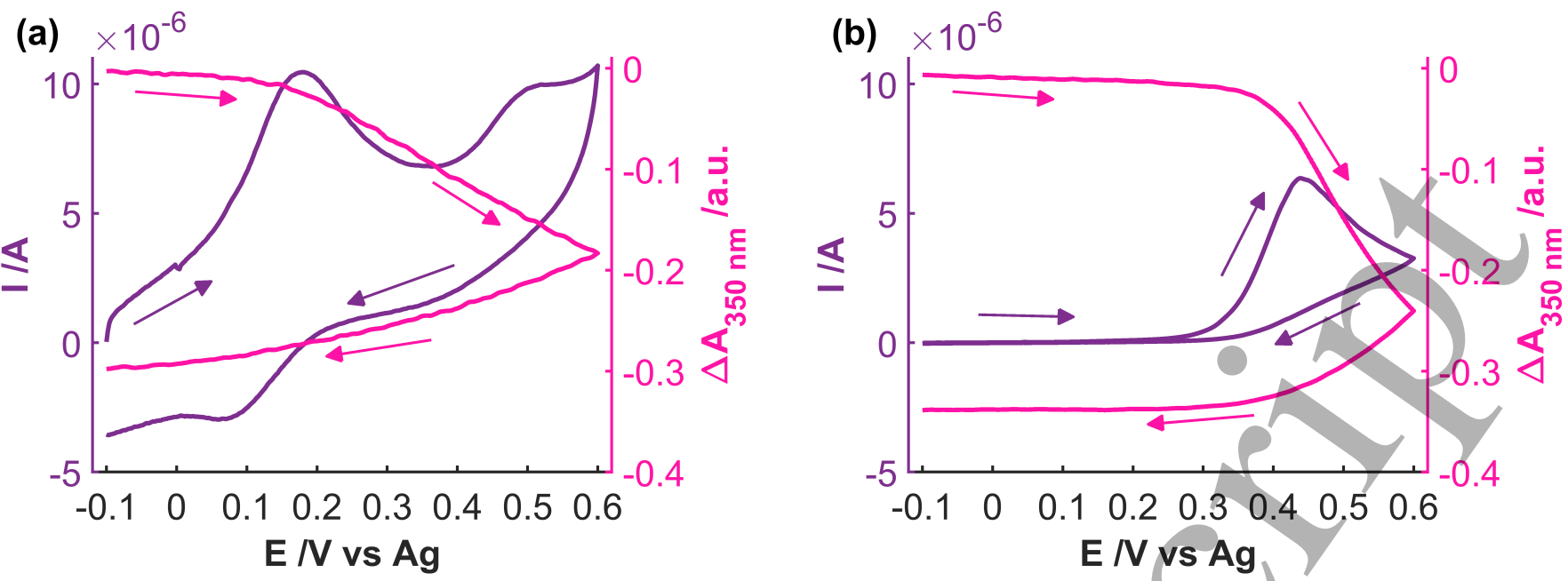

Figure 3 Cyclic voltammograms (purple lines) and cyclic voltabsorptograms at $350 \mathrm{~nm}$ (pink lines) collected at (a) EGO $\mathrm{DP}_{\mathrm{D}}$ and (b) EGO electrodes in 1.2 mM NADH in 0.1 M PBS (pH 7) and 0.1 M KCl. Potential was scanned between (a) $-0.10 \mathrm{~V}$ to $+0.60 \mathrm{~V}$ at $0.02 \mathrm{Vs}^{-1}$ and (b) $-0.10 \mathrm{~V}$ to $+0.60 \mathrm{~V}$ at $0.005 \mathrm{~V} \mathrm{~s}^{-1}$.

positive charges on the electrode surface, leading to electrostatic repulsion and possible desorption of unreacted DP molecules, which are positively charged at this $\mathrm{pH}$ value. Figure S7 in ESI also shows that the $\mathrm{EGO}_{\mathrm{DP}}$ coating was more stable than the MIX one, featuring a smaller lowering in current and achieving a stabilization not attained in the case of MIX.

Figure 2(a) shows the CV traces recorded after 20 scans in the pure electrolyte solution: $\mathrm{EGO}_{\mathrm{DP}}$ leaded to record more intense anodic and cathodic peaks with respect to MIX, confirming that the activation of oxidized moieties of EGO nanosheets by NHS/EDAC induces a higher number of DP molecules to be covalently linked to the carbon-based material.

Experiment reported in Figure 2(a) for $\mathrm{EGO}_{\mathrm{DP}}$ was performed on three different samples. These electrodes showed a very similar behavior: the relative standard deviation of the current peak intensity was $c a$. $1.8 \%$, indicating that $\mathrm{EGO}_{\mathrm{DP}}$ is a suitable for the use in disposable biosensors.

Figure 2(b) shows voltammograms obtained during the electrochemical oxidation of $0.5 \mathrm{mM} \mathrm{NADH}$ on the different materials (EGO, EGO $\mathrm{DP}_{\mathrm{DP}}$ and MIX). They were plotted after subtraction of the relevant electrochemical signal obtained in the absence of $\mathrm{NADH}$, in order to remove the contribution due to electroactive moieties on $\mathrm{EGO}_{\mathrm{DP}}$ and MIX (the original $\mathrm{CV}$ signals, collected in absence and in presence of NADH are reported in Figure S8 in ESI). The oxidation of $\mathrm{NADH}$ at EGO modified electrodes gives a well-defined peak at $c a .+0.50 \mathrm{~V}$, as expected from our previous results [7]. Conversely, both $\mathrm{EGO}_{\mathrm{DP}}$ and MIX modified electrodes also show an evident peak at $c a$. $+0.20 \mathrm{~V}$, which is higher in the case of $\mathrm{EGO}_{\mathrm{DP}}$. These results confirm that the intensity of the response obtained at $+0.20 \mathrm{~V}$ in the case of $\mathrm{NADH}$ oxidation is higher, the higher the number of 1,2dihydroxyphenyl moieties at EGO nanosheets (as indicated by the arrow in Figure 2(b)).

\subsection{UV-Vis absorption spectroelectrochemical study of NADH oxidation}

Current intensity recorded during a CV experiment always contains contributions of the capacitive current due to the polarization of the electrode surface and the background current. Conversely, optical absorbance can measure in a more selective manner changes in NADH concentration in the close proximity of the electrode surface, due to the oxidation process. For this reason, we decided to perform UV-Vis spectroelectrochemical experiments in parallel configuration to directly monitor the presence of chemical species produced or consumed during the electrochemical process $[43,44]$. This allowed us to study the role of 1,2dihydroxyphenyl residues covalently linked to EGO nanosheets.

In particular, spectra were collected to monitor the absorptometric changes in the NADH adsorption band at $c a$. $350 \mathrm{~nm}$, while changing the potential between -0.10 and $+0.60 \mathrm{~V}$. Conventional absorption spectroscopy performed in the bulk of the solution would not allow to follow in real time the NADH oxidation, due to the large volume of the solution and the limited diffusion of reacted species at macroscopic distance from the electrode. Instead, aligning the light beam parallel to the electrode surface and sampling the first $100 \mu \mathrm{m}$ of solution next to the electrode surface, it is possible to follow all spectroscopic changes that occur as consequence of the electrochemical reaction (see scheme in Figure S9 in ESI) [31,43]. 

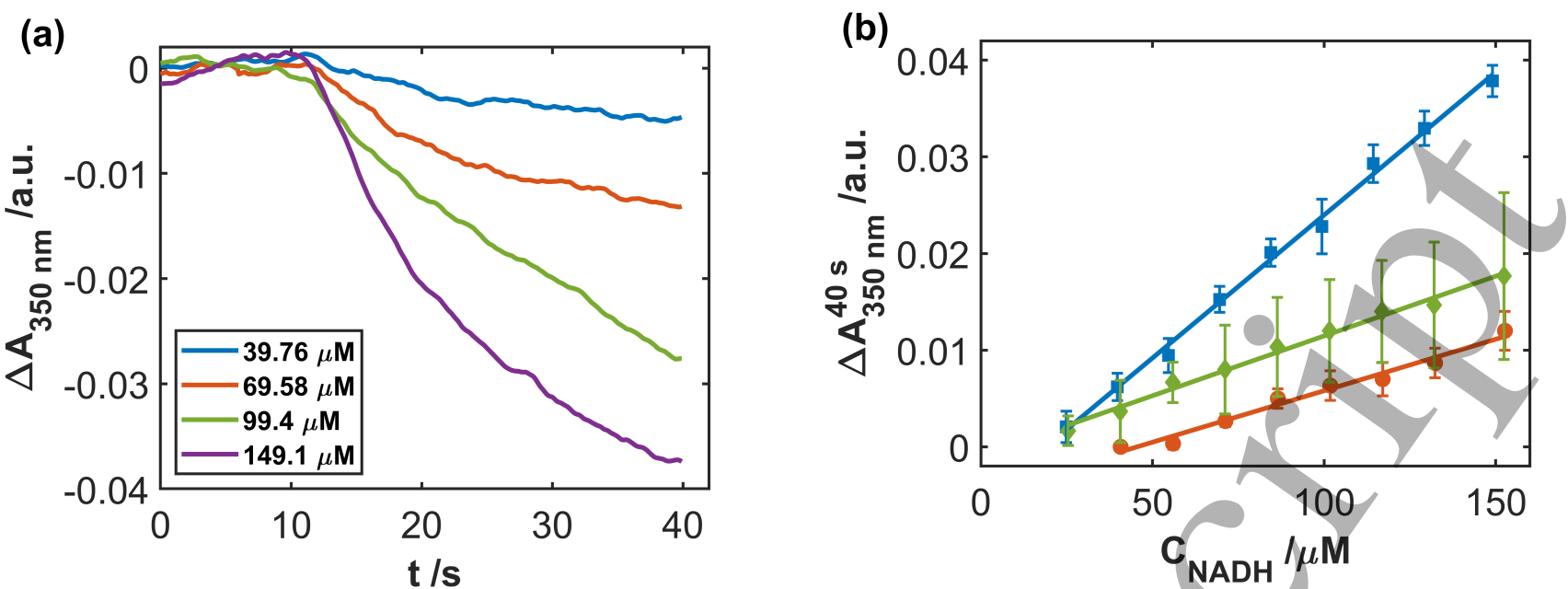

Figure 4. (a) Chronoabsorptograms recorded at $350 \mathrm{~nm}$ during chronoamperometric experiments in $0.1 \mathrm{M} \mathrm{PBS}$ and $0.1 \mathrm{KCl}$ solutions, containing NADH at different concentrations. $\mathrm{E}_{1}=-0.10 \mathrm{~V}, \mathrm{t}_{1}=10 \mathrm{~s}, \mathrm{E}_{2}=+0.26 \mathrm{~V}, \mathrm{t}_{2}=30 \mathrm{~s}$. (b) Relevant calibration plot of the absorbance at $350 \mathrm{~nm}$ measured $40 \mathrm{~s}$ after starting the experiment .Error bars are obtained from three consecutive calibrations performed on the same electrode; red line (EGO), blue line ( $\left.\mathrm{EGO}_{\mathrm{DP}}\right)$ and green line (MIX).

Figure 3 shows the superimposition of $i$ ) current measured during voltammetric oxidation of NADH (purple line) and ii) voltabsorptogram (optical absorption) of NADH at $350 \mathrm{~nm}$ (pink line).

The position of the anodic peaks at $+0.18 \mathrm{~V}$ and $+0.50 \mathrm{~V}$ in the $\mathrm{CV}$ traces reported in Figure 3(a) for $\mathrm{EGO}_{\mathrm{DP}}$ corresponds to that of the oxidation peaks already observed in Figure 2(b). The evolution of the UV-Vis absorption spectra during the $\mathrm{CV}$ experiment is shown in Figure S10 in ESI; for a better understanding of the figure, only the spectra registered during the anodic scan are displayed. It is observed a single absorption peak centred at $350 \mathrm{~nm}$, decreasing during the anodic sweep of the potential, due to NADH consumption with consequent formation of $\mathrm{NAD}^{+}$, which does not absorb in this spectral region. The evolution of a single absorption band within the whole anodic scan confirms that both electrochemical processes in Figure 3(a) are only ascribable to NADH oxidation to NAD

The combination of voltammetric (i vs. E) and of voltabsorptometric $\left(\Delta \mathrm{A}_{350} \mathrm{~nm} v \mathrm{vs}\right.$. E) traces in Figure 3(a) confirms that 1,2-dihydroxyphenyl residues can induce partial oxidation of NADH molecules at significantly lower potential values respect to the one observed for nonfunctionalized EGO (Figure 3(b)). Absorbance at $350 \mathrm{~nm}$ starts to decrease at around $+0.10 \mathrm{~V}$ in experiments performed on $\mathrm{EGO}_{\mathrm{DP}}$, whereas, the application of a potential as higher as $+0.40 \mathrm{~V}$ is necessary to begin NADH oxidation at pristine EGO. Since, as already stated by Figure 2(a), this is the same potential at which 1,2-hydroxyphenyl residues started to be oxidized in the pure electrolyte solution, we could conclude that NADH oxidation is mediated by chargetransfer reactions involving these organic moieties, introduced by functionalization of the EGO nanosheets with
DP molecules: once these redox reversible moieties are oxidized by application of suitable potential values, they can accept the electrons necessary for NADH oxidation [16]. Detection at such lower voltage gives a clear advantage in electrochemical sensors, avoiding simultaneous oxidation of different species in complex, multi-component biological matrices.

\subsection{EGO-based materials in the spectroelectrochemical determination of $\mathrm{NADH}$}

The performance of $\mathrm{EGO}_{\mathrm{DP}}$ compared to pristine $\mathrm{EGO}$ and MIX was tested as the sensing element for NADH detection by performing chronoabsorptometric measurements. In this case we measured absorbance changes at $350 \mathrm{~nm}$ while keeping the electrode at a fixed potential of $+0.26 \mathrm{~V}$ for $30 \mathrm{~s}$, i.e. at a potential value slightly higher than the oxidation peak, where the electrochemical process is almost limited by diffusion of NADH to the electrode surface and, as a consequence, the best sensitivity can be achieved. In these experiments, an initial potential of $-0.10 \mathrm{~V}$ was applied for $10 \mathrm{~s}$; no oxidation takes place in this interval time and there is no change in absorbance. Then, the potential was changed to $+0.26 \mathrm{~V}$, where the absorbance at $350 \mathrm{~nm}$ began to decrease with time due to oxidation of NADH. Figure 4(a) clearly shows that absorbance decrease obtained at $\mathrm{EGO}_{\mathrm{DP}}$ electrodes is proportional to the concentration of NADH in solution. A similar trend was also observed at pristine EGO and at MIX modified electrodes (data not shown).

The performance of the different materials was tested by repeating measurements in solutions at different NADH concentration, randomizing the measurements to exclude artefacts induced by possible memory effects. In addition, 
the calibration procedure was replicated three times on each electrode, to evaluate the repeatability of the sensor response. Figure 4(b) shows the calibration curves of optical absorption changes $(\Delta \mathrm{A})$ registered at $350 \mathrm{~nm}$ after applying the bias for $40 \mathrm{~s}$, as a function of the concentration of $\mathrm{NADH}$, averaged on three calibrations. A linear correlation between the two variables was observed in all measurements, indicating that all materials are suitable to be used as the reliable sensing element for enzyme-based sensors.

Comparison between the performance of the three materials was carried out comparing the Key Performance Indicators (KPIs) reported in Table 1, and calculated from responses shown in Figure 4(b) obtained by combining the results of three consecutive calibrations at the same electrode (see also Figure S11 and Table S2 in ESI to observe the three individual calibration responses). As already observed, pristine EGO had the worst sensitivity $\left(107 \mathrm{M}^{-1}\right)$ and the highest limit of detection ( $\mathrm{LOD}=2.79 \times 10^{-5} \mathrm{M}$ ) with respect to functionalized samples. The addition of DP molecules with redox active 1,2-dihydroxyphenyl residues in MIX allowed the material to perform better than pristine EGO, with a sensitivity of $124 \mathrm{M}^{-1}$ and $\mathrm{LOD}=2.14 \times 10^{-5} \mathrm{M}$; the improvement in sensitivity was about $150 \%$ vs. EGO.

$\mathrm{EGO}_{\mathrm{DP}}$ showed the best performance among all the samples tested, with the highest sensitivity $\left(298 \mathrm{M}^{-1}\right)$, an improvement of $\approx 180 \%$ vs. EGO, and lowest limit of detection $\left(\mathrm{LOD}=1.38 \times 10^{-5} \mathrm{M}\right)$. It also gave the best values of correlation coefficient $\left(\mathrm{R}^{2}\right)$ and of precision, represented by the value of standard deviation of the calibration line $\left(\mathrm{s}_{\mathrm{y} / \mathrm{x}}\right)$. Overall, Table 1 results demonstrate the advantages of adding 1,2-hydroxylphenyl moieties to EGO, especially if covalently bonded to EGO nanosheets. The best performance achieved with $\mathrm{EGO}_{\mathrm{DP}}$ can be ascribed to the poor stability of the organic residues in MIX, which gave more variable, nonoverlapping calibration plots (Figure S11 in ESI): sensitivity progressively decreased in consecutive analyses, indicating that the material is poorly stable when skipping the phase of chemical activation of oxidized residues of EGO nanosheets. On the contrary both EGO and $\mathrm{EGO}_{\mathrm{DP}}$ showed more stable responses in different measurements, demonstrating the better chemical stability of the overall coating on the electrode surface. The great repeatability observed at $\mathrm{EGO}_{\mathrm{DP}}$ based sensor is finally quantified by the standard deviation of the slope obtained from three calibrations performed on the same electrode $\left(\mathrm{RSD}_{\text {slope }}\right.$, Table 1$)$ in comparison to the values obtained for EGO and MIX. This result once more indicates that DP molecules can be more stably anchored to the EGO nanosheets by activation of oxidized residues with NHS/EDAC mixture; this approach makes the material suitable for effective electrochemical (bio)sensing.
Table 1. Analytical performance of the EGO, $\mathrm{EGO}_{\mathrm{DP}}$, and MIX modified electrodes defined on the basis of calibration plots reported in Figure 4.

\begin{tabular}{llll}
\hline & EGO & EGO $_{\text {DP }}$ & MIX \\
\hline sensitivity $\left(\mathrm{M}^{-1}\right)$ & $1.07 \times 10^{2}$ & $2.98 \times 10^{2}$ & $1.24 \times 10^{2}$ \\
\hline $\mathrm{R}^{2}$ & 0.950 & 0.989 & 0.677 \\
\hline $\mathrm{S}_{\mathrm{y} / \mathrm{x}}\left(\mathrm{M}^{-1}\right)$ & $0.99 \times 10^{-3}$ & $1.37 \times 10^{-3}$ & $4.15 \times 10^{-3}$ \\
\hline $\mathrm{RSD}_{\text {slope }}(\%)$ & 13.8 & 1.6 & 41.3 \\
\hline $\mathrm{LOD}(\mu \mathrm{M})$ & 27.9 & 13.8 & 21.4 \\
\hline & & & \\
& & & \\
4. Conclusion & & &
\end{tabular}

We have described a new approach for enhancing the performance of graphene oxide materials for the electrochemical oxidation of $\mathrm{NADH}$, by tailoring the $-\mathrm{OH}$ surface groups of EGO through covalent modification with dopamine. The use of $\mathrm{EGO}_{\mathrm{DP}}$ allows the improvement of the detection of NADH in terms of sensitivity and limit of detection with respect to pristine unfunctionalized EGO material and to the simple mixture of EGO and DP, namely MIX. The specific effect of 1,2-dihydroxyphenyl residues in the sensing capability was demonstrated by UV-Vis absorption spectroelectrochemical measurements. The construction of calibration plots from chronoabsorptograms revealed a significant higher sensitivity and lower LOD of $E_{\mathrm{DP}}$ functionalized samples with respect to pristine EGO and MIX.

The binding of DP to the nanosheets gives also a significant advantage in measurement stability, as compared to a simple mixture of the two components, confirming the effectiveness in the use of this material as the sensing element. The material can thus be used in electrochemical and spectroelectrochemical biosensors for the detection of NADH produced from a suitable NADH-dependent enzyme. As a very first test in this direction, lactate dehydrogenase was linked to the surface of the $\mathrm{EGO}_{\mathrm{DP}}$ using glutaraldehyde as a crosslinker [22], to obtain a sensor for lactic acid [20]. A linear correlation between the oxidation current and the concentration of analyte was observed in the 1-30 mM concentration range.

\section{Supporting Information}

ATR, SEM and XPS characterizations, CV data and spectroelectrochemical calibration plots. 


\section{Acknowledgements}

Authors acknowledge the financial support from the Spanish Ministerio de Economía y Competitividad (Grants CTQ2017-83935-R-AEI/FEDERUE), Ministerio de Ciencia, Innovación y Universidades (RED2018-102412-T) and Junta de Castilla y León (Grant BU297P18).

\section{References}

[1] Dragoman M and Dragoman D 2009 Prog. Quant. Electron. 33, 165.

[2] Geim AK 2009 Science 324, 1530

[3] Choi W, Indranil L, Raghunandan S and Kang YS 2010 Crit. Rev. Solid State Mater. Sci. 35, 52.

[4] Brownson DAC and Banks CE 2010 Analyst 135, 2768.

[5] Novoselov KS, Falko VI, Colombo L, Gellert PR, Schwab MG and Kim K 2012 Nature 490, 192.

[6] Hui KH, Pumera M and Bonanni A 2015 Chem. Eur. J. 21, 11793.

[7] Maccaferri G, Zanardi C, Xia ZY, Kovtun A, Liscio A, Terzi F, Palermo V and Seeber R 2017 Carbon 120, 165.

[8] Xia ZY, Giambastiani G, Christodoulou C, Nardi MV, Koch N, Treossi E, Bellani V, Pezzini S, Corticelli F, Morandi V, Zanelli A and Palermo V 2014 ChemPlusChem 79, 439.

[9] Naidek N, Zarbin AJG and Orth ES 2018 J. Polym. Sci., Part A: Polym. Chem. 56, 579.

[10] Tertiş M, Hosu O, Fritea L, Farcau C, Cernat A, Săndulescu R and Cristea C 2015 Electroanalysis 27, 638.

[11] Melucci M, Durso M, Zambianchi M, Treossi E, Xia ZY, Manet I, Giambastiani G, Ortolani L, Morandi V, De Angelis F and Palermo V 2012 J. Mater. Chem. 22, 18237.

[12] Maccaferri G, Terzi F, Xia Z, Vulcano F, Liscio A, Palermo $\mathrm{V}$ and Zanardi C 2019 Sens. Act. B, Chemical 281, 739.

[13] Uppal A and Gupta PK 2003 Biotechnol. Appl. Biochem. 37, 45.

[14] Mahmood MK, Akhtar H, Mir TA, Gurudatt NG, Chung S and Shim YB 2016 Biosens. Bioel. 85, 488.

[15] Molina BG, del Valle LJ, Turon P, Armelin E and Aleman C 2019 J. Phys Chem C 123, 22181.

[16] Katakis I and Domínguez E 1997 Mikrochim. Acta 126, 11.

[17] Radoi A and Compagnone D 2009 Bioelectrochemistry 76, 126.

[18] Mueller A 2005 Mini-Reviews in Medicinal Chemistry 5, 231.

[19] Li YW, Chen Y, Ma YH, Shi JG, Wang YX, Qi CH and Li QS 2014 Chin J Anal Chem 42, 759.

[20] Alama F, RoyChoudhurya S, Jalala AH, Umasankarb Y, Forouzanfara S, Aktera N, Bhansalia S and Palaa N 2018 Biosens. Bioel. 117, 818.

[21] Lin Y, Yu P, Hao J, Wang Y, Ohsaka T and Mao L 2014 Anal. Chem. 86, 3895

[22] Zanardi C, Ferrari L, Zanfrognini B, Pigani L, Terzi F, Cattini S, Rovati L and Seeber R 2013, Sensors 13, 4571.

[23] Zanardi C, Ferrari E, Pigani L, Arduini F and Seeber R 2005 Chemosensors 3, 118.

[24] Zhang M, Smith A and Gorski W 2004 Anal. Chem. 76, 5045.

[25] Li Z, Huang Y, Chen L, Qin X, Huang Z, Zhou Y, Meng Y, Li J, Huang S, Liu Y, Wang W, Xie Q and Yao S 2013 Sens. Act. B 181, 280.

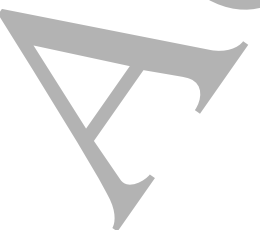

[26] Carlson BW and Miller LL 1985 J. Am. Chem. Soc. 107, 479.

[27] Gorton L and Domínguez E 2002 Rev Molecular Biotechnology 82, 371.

[28] Ghanem MA, Chrétien JM, Kilburn JD and Bartlett PN 2009 Bioelectrochemistry 76, 115.

[29] Xia Z, Leonardi F, Gobbi M, Liu Y, Bellani V, Liscio A, Kovtun A, Li R, Feng X, Orgiu E, Samorì P, Treossi E and Palermo V 2016 ACS Nano 10, 7125.

[30] Vacchi IA, Spinato C, Raya J, Bianco A and Ménard-Moyon C 2016 Nanoscale 8, 13714.

[31] Garoz-Ruiz J, Heras A and Colina A 2017 Anal. Chem. 89, 1815.

[32] Garoz-Ruiz J, Guillen-Posteguillo C, Heras A and Colina A 2018 Electrochem. Commun. 86, 12.

[33] Garoz-Ruiz J, Guillen-Posteguillo C, Colina A and Heras A 2019 Talanta 195, 815.

[34] Xia Y, Maccaferri G, Zanardi C, Christian M, Ortolani L, Morandi V, Bellani V, Kovtun A, Dell'Elce S, Candini A, Liscio A and Palermo V 2019 J. Phys. Chem. C 123, 1512215130.

[35] Kovtun A, Jones D, Dell'Elce S, Treossi E, Liscio A and Palermo V 2019 Carbon 143, 268.

[36] Bernsmann F, Ponche A, Ringwald C, Hemmerlè J, Raya J, Bechinger B, Voegel JC, Schaaf P, Ball V 2009 J. Phys. Chem. C 113, 8234.

[37] Clark MB, Gardella JA, Schultz TM, Patil DG and Salvati L 1990 Anal. Chem. 62, 949.

[38] Liu Y, Ai K and Lu L 2014 Chem. Rev. 114, 5057.

[39] Ryu JH, Messersmith PB and Lee H 2018 ACS Appl. Mater. Interfaces 10, 7523.

[40] Zhang X, Ho L, Fanny R and Samorì P 2018 Chem. Eur. J. 24, 18518 .

[41] Della Vecchia NF, Avolio R, Alfè M, Errico ME, Napolitano A and d'Ischia M 2013 Adv. Funct. Mater. 23, 1331.

[42] Posati T, Nocchetti M, Kovtun A, Donnadio A, Zambianchi M, Aluigi A, Capobianco ML, Corticelli F, Palermo V, Ruani G, Zamboni R, Navacchia ML and Melucci M 2019 ACS Omega 4, 4839.

[43] Orcajo O, Ventosa E, Martìnez A, Colina A, Heras A, Ruiz V and López-Palacios J 2006 J. Electroanal Chem 596, 95.

[44] Heras A, Vulcano F, Garoz-Ruiz J, Porcelli N, Terzi F, Colina A, Seeber R and Zanardi C 2019 Sensors 19, 518. 
